\title{
Diagnostic Role of Calretinin in Suspicious Cases of Hirschsprung's Disease
}

Sanjeev K. Singh ${ }^{1}$, Umesh K. Gupta ${ }^{2}$, Roopak Aggarwal ${ }^{1}$, Rafey A. Rahman ${ }^{2}$, Nand K. Gupta ${ }^{3}$, Vandana Verma $^{4}$

1. Pathology, Uttar Pradesh University of Medical Sciences, Saifai, Etawah, IND 2. Pediatric Surgery, Uttar Pradesh University of Medical Sciences, Saifai, Etawah, IND 3. Anatomy, Uttar Pradesh University of Medical Sciences, Saifai, Etawah, IND 4. Obstetrics and Gynecology, Uttar Pradesh University of Medical Sciences, Saifai, Etawah, IND

Corresponding author: Umesh K. Gupta, dukg9999@gmail.com

\section{Abstract \\ Background}

Hirschsprung's disease (HD) is a developmental disorder of the intrinsic component of the enteric nervous system. It is characterized by the absence of ganglion cells in the myenteric and submucosal plexus. Histopathological diagnosis becomes difficult many times due to submucosal ganglionic cells are not easily identifiable.

\section{Aims and objective}

The aim of this study was to examine the clinical and histopathological features of HD and to establish the utility of calretinin staining to diagnose the case of suspicious HD.

\section{Materials and methods}

After taking necessary informed consent, we studied 41 cases in which clinical suspicion of HD had been made, in a study duration of three years (July 2017-June 2020). Open biopsies were taken from spastic segment, transition zone and dilated segment. Histopathological diagnosis had been made in three categories: HD, no Hirschsprung's disease (NHD) and suspicion of HD. Post histopathological diagnosis calretinin immunohistochemistry (IHC) was applied to all cases and interpretations were noted.

\section{Results}

On the basis of histopathological findings, 25 cases were diagnosed as HD, nine cases were marked for suspicion for HD and seven cases as NHD. After evaluating calretinin IHC on the suspicious case, total of 30 cases were confirmed as HD while the remaining 11 cases were confirmed as NHD. Twenty-four patients of $\mathrm{HD}$ were males while the remaining six cases were females. The age of patients ranged from four days to 10 years. Median age six days while 22 patients were in the neonatal period.

Review began 02/10/2021 Review ended 02/11/2021 Published 02/16/2021

\section{() Copyright 2021}

Singh et al. This is an open access article distributed under the terms of the Creative Commons Attribution License CC-BY 4.0., which permits unrestricted use, distribution, and reproduction in any medium, provided the original author and source are credited.

\section{Conclusion}

Calretinin immunostaining is a useful modality in diagnosing suspicious cases of HD. Its results are easy to interpret by less experienced pathologist with accuracy.

Categories: Pathology, Pediatric Surgery, General Surgery

Keywords: immunohistochemistry, ganglion cells, congenital aganglionic megacolon, failure to thrive, constipation, functional intestinal obstruction, abdominal distension, aganglionosis

\section{Introduction}

Hirschsprung’s disease (HD) is also known as congenital aganglionic megacolon. HD is a developmental disorder of the intrinsic component of the enteric nervous system and it is characterized by the absence of ganglion cells in the myenteric and submucosal plexus [1]. These ganglion cells are responsible for normal peristalsis. Patients with HD usually present with functional intestinal obstruction at the level of aganglionosis. Most cases of HD involve the rectum or rectosigmoid region, but in $5 \%$ to $10 \%$ cases, the entire colon or part of small intestine may be involved [1]. Occurrence of HD is approximately 1 in 5,000 live births [2]. Males are more affected than females at a ratio around 4:1 [3,4].

In $80 \%$ to $90 \%$ cases of HD clinical features present soon after birth. HD should always be suspected when a new-born fails to pass meconium within 24 to 48 hours. Nearly $80 \%$ of patients show defecation problems along with delayed physical development, significant flatulence and emesis. While other HD patients usually don't show any problems until late childhood, when these patients present with chronic constipation, malnutrition and developmental delays. Occasionally patients may suffer from diarrhoeas, that 


\section{Cureus}

is, acute enteritis with a $30 \%$ fatality rate $[5,6]$.

Transmural bowel biopsies have been considered an important diagnostic tool. Suction rectal biopsy is recommended in most centers as an outdoor procedure but its cost of instrument is a limiting factor for use. On hematoxylin and eosin (H\&E) staining, lack of ganglion cells in the submucosal or intramuscular nerve plexus of the intestinal wall and hypertrophic nerve fibers and trunks are diagnostic of HD [7]. On immunohistochemistry (IHC) of patients who do not have HD, intrinsic nerves of muscularis mucosae and lamina propria show calretinin positivity. While in HD, this staining pattern is lost [8].

\section{Materials And Methods}

This study was a retrospective observational study conducted in the Department of Pathology in collaboration with the Department of Pediatric Surgery, Uttar Pradesh University of Medical Sciences (UPUMS), Saifai, Etawah, UP for three years (July 2017 to June 2020). Our institute is a tertiary health care centre of northern India, primarily providing health care to the rural and semi-urban population of this region. Ethical clearance was obtained from the institutional ethical committee. Necessary informed consent was obtained from included patient's parents.

Total 41 cases with the clinical presentation of neonatal intestinal obstruction to chronic progressive constipation in older children and whose radiological features (on X-ray and barium/contrast enema) were consistent with HD (e.g., paucity of air in pelvis, reversal of rectosigmoid index) were included in the study (Figure $1 \mathrm{~A}, \mathrm{~B}$ ). The intra-operative finding of dilated proximal bowel loop and spastic aganglionic segment were seen in all patients of this study (Figure 1C). All patients were initially managed with diverting leveling colostomy, that is, taking biopsy from the colostomy site. Multiple biopsies were taken from spastic segment, transition zone and proximal dilated segment.


\section{FIGURE 1: Image showing radiological and surgical features of HD.}

(A) Plain X-ray: paucity of air in the pelvic cavity (white arrow); (B) Contrast enema: sigmoid colon (star shape) is more dilated than rectum (reversal of rectosigmoid index) and 24-hour delayed film holding of dye in bowel (hexagonal shape); (C) intra-operative dilated sigmoid colon; (D) intra-operative retro rectal (white arrow retro rectal space) pull through (black arrow pull through bowel). HD: Hirschsprung's disease. 


\section{Cureus}

The biopsy specimens were received in $10 \%$ formalin. After tissue processing, sections were submitted for microscopic examination. Multiple H\&E-stained sections were examined and on the basis of presence or absence of ganglion cells, these were categorized into three diagnosis: HD, NHD and suspicion of HD (Table 1). Thereafter Calretinin immunostaining was applied (monoclonal rabbit IgG, Thermo Fisher Scientific, Cheshire, UK) on all the biopsy samples. These IHC slides were examined by two pathologists separately (Kappa value $=1$ ). On examination of calretinin IHC slides, section from aganglionic segments (spastic) showed negative calretinin expression, while positive calretinin expression was seen in normal colon section (Figure 2A-D). After histopathology reports, patients with confirmed HD were managed by a definitive pullthrough procedure (Figure 1D) while in NHD patient's colostomy closure was done.

\begin{tabular}{|l|l|l|}
\hline Categories & Histologic features (H\&E) & IHC features (calretinin) \\
\hline HD & $\begin{array}{l}\text { No ganglion cells (in spastic region), nerve } \\
\text { hypertrophy }\end{array}$ & Absence of calretinin expression in nerve fibres \\
NHD & $\begin{array}{l}\text { Ganglion cells present, no nerve } \\
\text { hypertrophy }\end{array}$ & $\begin{array}{l}\text { Staining present in submucosal nerve plexus, or in muscularis mucosa } \\
\text { or in lamina propria }\end{array}$ \\
$\begin{array}{l}\text { Suspicious } \\
\text { HD }\end{array}$ & No ganglion cells, no nerve hypertrophy & No suspicion after evaluation of calretinin expression \\
\hline
\end{tabular}

\section{TABLE 1: Histopathological and IHC criteria of classifying Hirschsprung's disease.}

H\&E: hematoxylin and eosin; IHC: immunohistochemistry; HD: Hirschsprung's disease; NHD: non-Hirschsprung's disease.


\section{FIGURE 2: Microphotograph of histopathological examination and Immunohistochemistry finding of HD.}

(A) Micrograph 400X, H\&E stain, ganglion cells (shown by black arrow) seen in NHD cases; (B) micrograph 100X, calretinin stain, ganglion cells (shown by white arrow) seen in NHD cases; (C) micrograph 400X, calretinin stain, ganglion cells (shown by white arrow) seen in suspected HD cases; (D) micrograph 100X, 


\section{Cureus}

calretinin stain ganglion cells not seen in suspected HD cases. HD: Hirschsprung's disease; NHD: nonHirschsprung's disease; H\&E: hematoxylin and eosin.

Only cases with adequate biopsies samples were included in this study. Cases with inadequate biopsies, inadequate clinical details and autolysed specimen were excluded from the study.

\section{Results}

During the study period of three years, total of 41 adequate biopsies were received from cases with clinical suspicion of HD. Out of these 41 cases, 30 cases were diagnosed as HD, on the basis of histopathological examination and calretinin IHC. The age of confirmed HD patients ranged from four days to 10 years (median age was six days). Twenty-two patients were in the neonatal period (Table 2). Constipation was the most common presenting symptom (96.7\%) in confirmed HD cases in the study. Intestinal obstruction with or without constipation was the second most common symptom (80\%) in HD patients. Only $23.3 \%$ of patients were presented with failure to thrive (Table 3).

\begin{tabular}{|c|c|c|}
\hline Age of the patient (confirmed HD) & Number of patients & Percentage \\
\hline $0-28$ days & 22 & 73.33 \\
\hline $1-12$ months & 05 & 16.67 \\
\hline$>1$ year & 03 & 10.00 \\
\hline Total & 30 & \\
\hline
\end{tabular}

TABLE 2: Age group of confirmed Hirschsprung's disease (HD) patients.

\begin{tabular}{|l|l|l|}
\hline \hline Clinical presentation & Number of cases & Percentage \\
\hline Constipation* & 29 & 96.7 \\
\hline Intestinal obstruction & 24 & 80.0 \\
\hline Failure to thrive & 07 & 23.3 \\
\hline
\end{tabular}

TABLE 3: Distribution of confirmed HD $(n=30)$ cases according to clinical presentation.

*Failure to pass meconium or infrequent passage of stool. HD: Hirschsprung's disease.

On the basis of histopathology reports (H\&E stained sections) out of the 41 cases, 25 (60.97\%) cases were diagnosed as HD, 9 (21.95) cases as suspicious HD and 7 (17.07\%) cases as negative for HD. IHC for calretinin was applied on all 41 cases (Table 4). After application of calretinin IHC, out of 41 cases, 30 (73.17\%) cases were confirmed as HD while the remaining 11 (26.82\%) cases were confirmed as NHD, hence all suspicious HD cases had been confirmed and categorized in HD and NHD groups (Table 4). Out of total 30 confirmed cases of HD, 24 (80\%) patients were males while the remaining 6 (20\%) cases were females and $\mathrm{M} / \mathrm{F}$ ratio was 4:1. Twenty-two of the confirmed HD patients were neonates ( $<1$ month age). Twenty-nine cases out of 30 confirmed HD cases were short segment HD. In our study sensitivity of calretinin positivity for ganglion cells was $100 \%$, specificity was $68.75 \%$, while positive and negative predictive values were $85.33 \%$ and $100 \%$, respectively. Same findings were reconfirmed in tissue resected during the definitive procedure. 


\section{Cureus}

\begin{tabular}{|c|c|c|}
\hline Categories & Based on H\&E & Based on calretinin IHC \\
\hline HD & 25 & 30 \\
\hline NHD & 7 & 11 \\
\hline Suspicious HD & 9 & 0 \\
\hline Total & 41 & 41 \\
\hline
\end{tabular}

TABLE 4: Hirschsprung's disease diagnosis on basis of H\&E staining and calretinin IHC.

H\&E: hematoxylin and eosin; IHC: immunohistochemistry; HD: Hirschsprung's disease; NHD: non-Hirschsprung's disease.

\section{Discussion}

$\mathrm{HD}$ is one of the commonest causes of functional intestinal obstruction in children. Early diagnosis and timely surgical intervention are important to prevent disease associated with morbidity and mortality [9]. In our study, the majority of patients were males and male to female ratio (M/F) was 4:1. While many studies showed strong male predominance for example Ziad et al., found male/female ratio of 5.8/1 among HD patients [10]. Majority of HD patients (80\%-90\%) are diagnosed in the neonatal period [11]. In our study, 22 cases out of 30 (73.3\%) were neonates. While in the study by Ziad et al. [10], they found $57 \%$ of their HD patients were neonates. In a study by Archibong, late presentation of patient was attributed to ignorance and poverty [9]. Clinical presentation of HD depends on the length of the aganglionic segment and severity of disease. Chief clinical presentations are delayed passage of meconium, intestinal obstruction, intestinal perforation, enterocolitis and constipation [12]. Intestinal obstruction is usually associated with distended abdomen, bilious vomiting, fever, dehydration, lethargy and not passing meconium. While in perforation, features of perforation peritonitis predominates, and in neonates with this condition, colon must be thoroughly examined and appropriate biopsies should have been taken [12]. In our study, among confirmed HD cases, most of the cases (96.7\%) presented with constipation (failure to pass meconium in neonates and infrequent passage of stools in older patients) and second most common (80\%) clinical presentation was intestinal obstruction.

About $70 \%$ to $75 \%$ of HD can be diagnosed with barium or contrast enemas. However classical radiological features are absent in about $25 \%$ of neonates [13]. Biopsy is the gold standard for the diagnosis of HD [12]. Suction rectal biopsy (SRB) is an important tool available for outpatient procedure. In our study, we performed full-thickness biopsy, because patients came in sick condition and urgent laparotomy was necessary. Required instruments essential for SRB and facility for study of acetylcholinesterase (AChE) were not available in our institute. We explored all the patients on the basis of clinical history and radiological feature of this study and took multiple full-thickness biopsies. Surgery had been performed in all the patients in our study. Colostomy with multiple biopsies was done as primary procedure. After confirmation of diagnosis by histopathology and IHC, definitive pull-through and/or colostomy closure was done. Studies showed that Duhamel's endorectal pull-through should be the preferred surgical treatment with least complications [14,15].

In histopathological examination, lack of ganglion cells in submucosal nerve plexus is required for diagnosis of HD. In our study after evaluation of H\&E-stained slides, we confirmed 25 cases as HD and seven cases as $\mathrm{NHD}$, while nine cases termed as suspected HD. We used calretinin immunostaining in all cases. In our study all NHD cases expressed calretinin positivity, while all HD cases (30 cases) showed negative for calretinin. In a study, Zuikova et al. [16] stated that calretinin IHC is easier to interpret than AChE. Barshack et al. [8] also concluded that aganglionic segments (spastic) shows negative calretinin expression, while positive calretinin expression is seen in normal colon section. Maldyk et al. [17] also confirmed in their study that calretinin expression was positive in all rectal biopsies with ganglionic cells, while expression was negative in all aganglionic segments. In our study sensitivity of calretinin positivity for ganglion cells was $100 \%$, specificity was $68.75 \%$, while positive and negative predictive values were $85.33 \%$ and $100 \%$, respectively. Mukhopadhyay et al. [18] stated that sensitivity of calretinin positivity for ganglion cells was $100 \%$, specificity was $97.44 \%$, while positive and negative predictive values were $84.62 \%$ and $100 \%$, respectively.

In a study by Gonzalo and Plesec [19], all patients without HD had calretinin positive nerve fibres in lamina propria or muscularis mucosa and all patients with HD showed no calretinin staining of nerve fibres in the same regions. Guinard-Samuel et al. [20] also noticed 12 cases as suspicious for HD And these suspicious cases were accurately diagnosed by calretinin immunohistochemistry. In the present study, after evaluating calretinin IHC on suspicious cases for HD, we confirmed HD in five of these suspicious cases, because of negative calretinin expression. Rest of the four cases showed calretinin positivity, hence confirmed as NHD.

The limitation of our study is a relatively small sample size. In the study duration of three years, we 
diagnosed only 30 confirmed HD cases. Further studies with larger sample are desirable.

\section{Conclusions}

Our study showed male predominance in confirmed HD cases. Constipation and intestinal obstruction were the most common clinical presentation in these cases. The study also concludes that calretinin immunostaining is very useful to diagnose suspected HD cases. Calretinin IHC should be routinely used in all suspicious cases of HD.

\section{Additional Information \\ Disclosures}

Human subjects: Consent was obtained or waived by all participants in this study. Ethical committee Uttar Pradesh university of medical sciences, saifai issued approval 111/2020-21. Animal subjects: All authors have confirmed that this study did not involve animal subjects or tissue. Conflicts of interest: In compliance with the ICMJE uniform disclosure form, all authors declare the following: Payment/services info: All authors have declared that no financial support was received from any organization for the submitted work. Financial relationships: All authors have declared that they have no financial relationships at present or within the previous three years with any organizations that might have an interest in the submitted work. Other relationships: All authors have declared that there are no other relationships or activities that could appear to have influenced the submitted work.

\section{References}

1. Langer JC: Hirschsprung’s disease. In: Pediatric Surgery, pp 1265-1278 . Coran AG, Adzick NS, Krummel TM, Laberge JM, Caldamone A, Shamberger R (ed): Elsevier Saunders, Philadelphia, PA; 2012. 10.1016/B978-0323-07255-7.00101-X

2. Kenny SE, Tam PK, Garcia-Barcelo M: Hirschsprung disease. Semin Pediatr Surg. 2010, 19:194-200. 10.1053/j.sempedsurg.2010.03.004

3. de Lorijn F, Boeckxstaens GE, Benninga MA: Symptomatology, pathophysiology, diagnostic workup and 24 treatment of Hirschsprung disease in infancy and childhood. Curr Gastroenterol Rep. 2007, 9:245-253. 10.1007/s11894-007-0026-z

4. Martucciello G: Hirschsprung's disease, one of the most difficult diagnoses in pediatric surgery: a review of the problems from clinical practice to the bench. Eur J Pediatr Surg. 2008, 18:140-149. 10.1055/s-20081038625

5. Holschneider AM, Puri P: Hirschsprung’s disease and allied disorders, pp 110-114 . Springer, Berlin; 2008. 10.1007/978-3-540-33935-9

6. Khan AR, Vujanic GM, Huddart S: The constipated child: how likely is Hirschsprung’s disease? . Pediatr Surg Int. 2003, 19:439-442. 10.1007/s00383-002-0934-9

7. Harjai MM: Hirschsprung disease: revisited. J Postgrad Med. 2000, 46:52-54.

8. Barshack I, Fridman E, Goldberg I, Chowers Y, Kopolovic J: The loss of calretinin expression indicates aganglionosis in Hirschsprung's disease. J Clin Pathol. 2004, 57:712-716. 10.1136/jcp.2004.016030

9. Mabula JB, Kayange NM, Manyama M, Chandika AB, Rambau PF, Chalya PL: Hirschsprung's disease in children: a five year experience at a university teaching hospital in northwestern Tanzania. BMC Res Notes. 2014, 7:410. 10.1186/1756-0500-7-410

10. Ziad F, Katchy KC, Ramadan SA, Alexander S, Kumar S: Clinicopathological features in 102 cases of Hirschsprung's disease. Ann Saudi Med. 2006, 26:200-204. 10.5144/0256-4947.2006.200

11. Puri P: Newborn Surgery, 3rd Edn. pp 554-565. CRC Press, London; 2011. 10.1201/b13478

12. Bhatnagar SN: Hirschsprung's disease in newborns . J Neonatal Surg. 2013, 2:51. 10.47338/jns.v2.63

13. Smith GH, Cass D: Infantile Hirschsprung’s disease- Is barium enema useful? . Pediatr Surg Int. 1991, 6:318321. 10.1007/BF00178647

14. McCready RA, Beart RW Jr: Adult Hirschsprung's disease: results of surgical treatment at Mayo Clinic . Dis Colon Rectum. 1980, 23:401-407. 10.1007/BF02586788

15. Elliot MS, Todd IP: Adult Hirschsprung’s disease: results of the Duhamel procedure. Br J Surg. 1985, 72:884885. 10.1002/bjs.1800721112

16. Zuikova V, Franckevica I, Strumfa I, Melderis I: Immunohistochemical diagnosis of Hirschsprung’s disease and allied disorders. Acta Chirurgica Latviensis. 2015, 15:50-57. 10.1515/chilat-2016-0010

17. Maldyk J, Rybczynska J, Piotrowski D, Kozielski R: Evaluation of calretinin immunohistochemistry as an additional tool in confirming the diagnosis of Hirschsprung’s disease. Pol J Pathol. 2014, 65:34-39. 10.5114/pip.2014.42667

18. Mukhopadhyay B, Mukhopadhyay M, Mondal KC, Sengupta M, Paul A: Hirschsprung’s disease in neonates with special reference to Calretinin Immunohistochemistry. J Clin Diagn Res. 2015, 9:EC06-EC09. 10.7860/JCDR/2015/12470.6180

19. Gonzalo DH, Plesec T: Hirschsprung disease and use of calretinin in inadequate rectal suction biopsies . Arch Pathol Lab Med. 2013, 137:1099-1102. 10.5858/arpa.2012-0220-OA

20. Guinard-Samuel V, Bonnard A, De Lagausie P, et al.: Calretinin immunohistochemistry: a simple and efficient tool to diagnose Hirschsprung disease. Modern Pathol. 2009, 22:1379-1384.

10.1038/modpathol.2009.110 